\title{
Mitteilungen der Deutschen Gesellschaft für Rechtsmedizin
}

\section{Nachruf auf Professor Dr. rer. nat. Rudhard Klaus Müller}

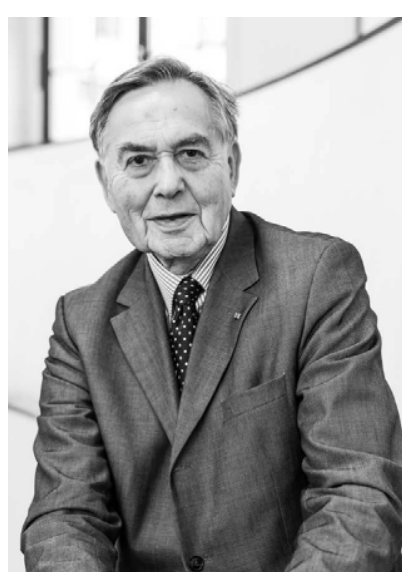

Abb. 1 \ Prof. Klaus Müller. (C) Fotoausschnitt: „30 Jahre PGS Toxikologie und Umweltschutz Gestern und Heute", 2018, ISBN 978-3-930865-09-3)
Klaus Müller (siehe $\bullet$ Abb. 1) verstarb im Alter von 85 Jahren im Kreise seiner Familie in Leipzig. Noch im Frühjahr konnten wir ihn und seine Frau im ehemaligen Anwesen in Waldsteinburg zum sächsischen Kaffeetrinken mit frischen Windbeuteln besuchen. Dabei haben wir uns angeregt über seine Interessen zur Anatomie, über die Musik und die Umzugspläne zurück in die Stadt unterhalten. Es war ein herzlicher Nachmittag bei dem wir uns trotz der Corona-Pandemie mit einem für ihn typischen kräftigen Händedruck verabschiedeten. Nachfolgend sei an die wichtigsten

Stationen seines vielfältigen Schaffens erinnert:

Klaus Müller wurde am 20.08.1936 in Glauchau, gelegen an der Zwickauer Mulde, geboren. Nach der Gymnasialzeit an der „Erweiterten Oberschule" Georgius-Agricola und einer Relegierung nach politischer Anklage 1953 legte er 1954 das Abitur ab und hatte die Absicht, Chemie zu studieren. Eine Bewerbung dafür an der Universität Leipzig war zunächst aussichtslos: Chemie galt damals als überlaufendstes Fach. Dazu waren in den zwei Jahren zuvor wegen des Staatsvorhabens „Chemie bringt Brot, Wohlstand und Schönheit" für die zu gründenden Forschungszentren hunderte Chemiestudenten immatrikuliert worden und hatten alles überfüllt. Als Angestelltenkind hatte er auch mit der Abiturnote 1 keine Chance. Die Bewerbung an der Uni Halle wurde sofort an die gerade gegründete Technische Hochschule Leuna-Merseburg weitergeleitet. Da die neue Hochschule vorerst nur auf dem Papier bestand, begann das Studium in einem alten Gebäude in Halle, mit der Immatrikulation in Leuna und der Grundsteinlegung für den Hochschulbau in Merseburg.

Wegen der nach dem Studium beabsichtigten Orientierung auf ein medizinisches Grenzgebiet beantragte Klaus Müller den Hochschulwechsel nach Leipzig und kam 1955 an die Alma Mater Lipsiensis. Ab 1956 studierte er parallel auch Humanmedizin bis 1962. Nach erfolgreichem Staatsexamen in Chemie 1960 arbeitete er als wissenschaftlicher Mitarbeiter am Institut für Gerichtliche Medizin und Kriminalistik zunächst unter der kommissarischen Leitung von Professor Otto Prokop und ab 1962 unter dem Ordinariat von Professor Wolfgang Dürwald bis 1989.

Rechtsmedizin $2022 \cdot 32: 77-80$

https://doi.org/10.1007/s00194-021-00551-z

(C) The Author(s), under exclusive licence to Springer Medizin Verlag $\mathrm{GmbH}$, ein Teil von Springer Nature 2021

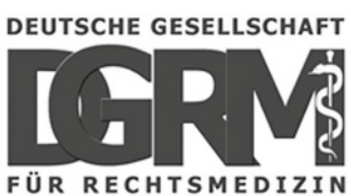

Verantwortlich für den Inhalt der Mitteilungsseiten der DGRM

Prof. Dr. Silke Grabherr

Institut für Rechtsmedizin

Lausanne-Genf (CURML)

Chemin de la Vulliette 4

$\mathrm{CH}$ - 1000 Lausanne 25

silke.grabherr@chuv.ch

Die doppelte Qualifikation in Chemie und Medizin befähigte ihn im facettenreichen Fachgebiet der Gerichtsmedizin besonders in der Abteilung für toxikologische Chemie an der Entwicklung dieses Grenzgebietes zwischen analytischer Chemie und Toxikologie mitzuarbeiten. 1965 promovierte Klaus Müller zum Dr. rer. nat. mit dem Thema „Beiträge zur Systematisierung der qualitativen Analyse in der gerichtlichen Chemie". 12 Jahre später habilitierte er sich an der Akademie der Wissenschaften der DDR für das Fach Toxikologie mit den Ergebnissen seiner anwendungsorientierten Forschungen zu ausgewählten Analyseverfahren bei akuten Intoxikationen. Als Nicht-Mitglied in der SED brauchte er 5 Jahre bis zur Ernennung zum Hochschuldozenten für Forensischen Toxikologie. Erst 1989, unmittelbar vor der politischen Wende, erfolgte die Ernennung zum außerordentlichen Professor und 1992 schließlich die Berufung auf die Professur für Forensische Toxikologie an der Medizinischen Fakultät der Universität Leipzig. Zuvor, 1991, wurde Klaus Müller gebeten, im Nebenamt das de facto abgewickelte Dopinglabor Kreischa neu zu etablieren und als künftiges Institut für Dopinganalytik und Sportbiochemie Dresden zu leiten. Sein unermüdliches wissenschaftliches und sportpolitisches Engagement führte zu leitenden Positionen im Rahmen der Antidopingkonvention des Europarats und zur Mitarbeit in der Welt-Antidopingagentur WADA Montreal von deren Gründung im Jahre 2000 an bis 2007. Im gleichen Jahr wurde er in den ehrenamtlichen Vorstand der deutschen Nationalen Anti-Doping Agentur (NADA) berufen bis zu deren Umstrukturierung zur hauptberuflichen Geschäftsleitung. Diese Aufgaben mit vollem Einsatz zu erfüllen bedeutete für Klaus Müller 14 Jahre einen weitgehenden Verzicht auf Freizeit und ein selbstbestimmtes Zeitregime.

In den vielen Jahren seines beruflichen Schaffens auch als Emeritus hat Klaus Müller mehr als 400 Publikationen veröffentlicht, darunter mehrere Bücher und zahlreiche Sammelbände. Sein über 800 Seiten starkes Werk „Toxikologisch-chemischen Analyse" übergab er dem aktuellen Leiter der Rechtsmedizin zu seiner Berufung nach Leipzig.

Es dient ihm immer wieder als wertvolles Nachschlagewerk zu speziellen analytischen Fragestellungen.

Das umfangreiche Werk erschien bereits 1976 und war für viele Jahre die Arbeitsgrundlage der forensisch toxikologischen Analytik. Nicht nur im Rahmen der heute bezeichneten Standardar- 
beitsanweisungen und gelenkten Dokumente hatte Klaus Müller schon im Vorfeld fortschrittliche Vorgaben, sondern auch auf dem Gebiet der Wissensorganisation. So initiierte er ein Informationssystem für die Toxikologische Chemie, ISTOC. Diese Literaturdaten wurden an viele forensisch toxikologische Einrichtungen versandt. Gebündelt wurden die Aktivitäten durch die Arbeitsgemeinschaft Toxikologische Chemie der Gesellschaft für Gerichtliche Medizin der DDR, AGTC, die er in den Jahren von 1967 bis 1990 geleitet hat.

Besondere Verdienste erwarb sich Klaus Müller auch bei der Qualifizierung des wissenschaftlichen Nachwuchses. Er erkannte frühzeitig, dass es in der besonderen Verantwortung der heute in den Fachgesellschaften Aktiven liegt, qualifizierte Fachtoxikologen auszubilden und gründete 1987 den noch heute sehr erfolgreichen Studiengang Toxikologie und Umweltschutz an unserer Universität. Ein großer Teil der heute in Deutschland und darüber hinaus maßgeblich tätigen Toxikologen sind Absolventen dieses immer mit ca. 80 Studenten gut besuchten Aufbaustudiums.

In den letzten Jahren wirkte Klaus Müller als ehrenamtlicher Vorsitzender der Landessektion Sachsen des Bundes gegen Alkohol und Drogen im Straßenverkehr. Unter den vielen Ehrungen, die Klaus Müller erhalten hat, möchten wir insbesondere den Leibniz-Preis der Universität Leipzig, die Richard-Kockel-Medaille der Gesellschaft für Gerichtliche Medizin der DDR, das Bundesverdienstkreuz am Bande und den Alan Curry - Award, die höchste Auszeichnung der International Association of Forensic Toxicologists TIAFT erwähnen.

Das Oeuvre von Klaus Müller war erstaunlich vielseitig. Er spielte Klavier und Orgel, wartete literarisch mit seinen Limericks und besonderen Aktivitäten und Ämtern im Rotary Club Leipzig auf und sammelte neben Grafiken auch Oldtimer und Nussknacker.

Unsere letzte wissenschaftliche Würdigung erfuhr Klaus Müller anlässlich seines 80. Geburtstages im September 2016 im Rahmen derTagung „Forensische Toxikologie im Umfeld“, der 18. Tagung der Sächsischen Institute für Rechtsmedizin und des LKA Sachsen. Der Jubilar ergriff zum Abschluss das Wort und lies sein wissenschaftliches Werk Revue passieren. Der Abschluss war gekrönt mit seinem Orgelspiel in der Petri-Kirche zu Leipzig an der Jahn-Orgel der ehemaligen Universitätskirche.

Lieber Freund Klaus, wir vermissen dich!

Wir werden dein Vermächtnis ehren und fortsetzen.

\section{J. Dreßler und A. Graefe}

Leipzig

\section{Nachruf zu \\ Prof. Dr. Claus Henßge}

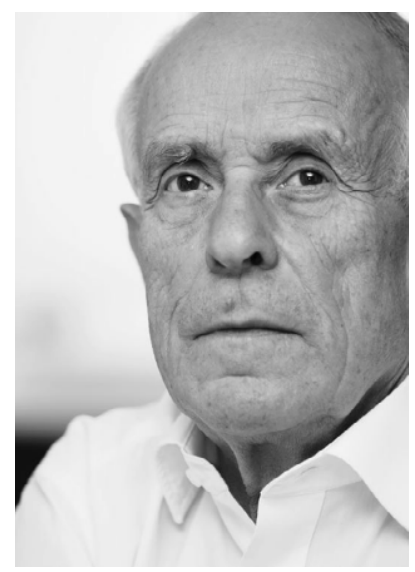

\author{
Nachtrag zu https:// \\ doi.org/10.1007/ \\ s00194-021-00541-1
}

Leider wurde in der letzten Ausgabe der Mitteilungsseiten versäumt, die korrekte Quelle (๔ Timo Bobert, Berlin) des Porträtfotos anzugeben. Wir bitten, dies zu entschuldigen und drucken Foto und Quelle hier noch einmal ab.

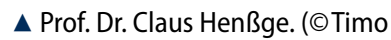
Bobert, Berlin)

\title{
Gedenkfeier zum 100. Geburtstag von Otto Prokop
}

\author{
Berlin, 29. September 2021
}

Am 29.09.2021 wurden in einer Feierstunde die wissenschaftlichen Leistungen des Berliner Gerichtsmediziners Otto Prokop (19212009) gewürdigt (siehe G. Geserick \& I. Wirth „Zum 100. Geburtstag von Otto Prokop (1921-2009),' Rechtsmedizin 2021 31:299-305). Eigens hierfür wurden die Pforten des ehemaligen Instituts für Gerichtliche Medizin der Humboldt-Universität zu Berlin in der Hannoverschen Straße 6 geöffnet. An diesem Ort verbrachte der Jubilar fast 50 Jahre seines Lebens, davon 30 Jahre als Institutsdirektor und Lehrstuhlinhaber. 12 Jahre lang bewohnte Familie Prokop eine Dienstwohnung im Institut. Heute beherbergt das Gebäude das Institut für

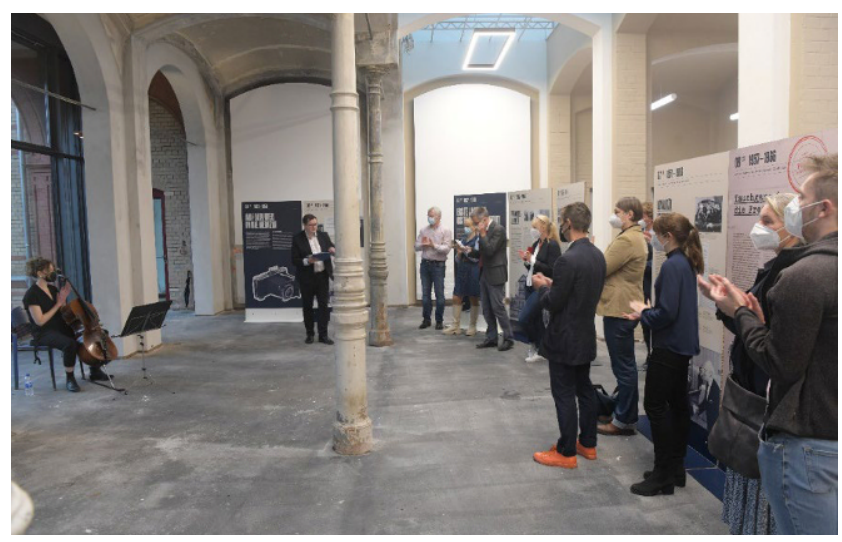

Abb. 2 \ Gedenkfeier in der Charité. (@ Charité/Wiebke Peitz) 
Katholische Theologie der Humboldt-Universität sowie das Berliner Institut für Islamische Theologie.

In dem einst als polizeiliches Leichenschauhaus genutzten Mitteltrakt des Gebäudes, der derzeit aufwändig saniert und zu einer Ausstellungsfläche umgebaut wird, wurde anlassbezogen die Ausstellung "Sezierte Wahrheiten - Otto Prokop und sein Institut für Gerichtliche Medizin im geteilten Berlin“ installiert.

Unter den geladenen Gästen waren neben Vertretern der Humboldt-Universität und der Charité Mitglieder der Familie Prokop und sein Schüler und Nachfolger im Amt Prof. Dr. med. Gunther Geserick (*1936), der in bewegenden lobenden aber auch leisen kritischen Worten an seinen akademischen Lehrer erinnerte.

Eingerahmt wurden die Redebeiträge in die Allemande und Courante aus der Cellosuite Nr. 4 in Es-Dur von Johann Sebastian Bach eine instrumentale Premiere am historischen Ort (siehe $\bullet$ Abb. 2).

\section{S. Hartwig}

Berlin

\section{Bericht von der Delegierten- konferenz (DK) der Arbeitsgemeinschaft der Wissenschaftlichen Medizi- nischen Fachgesellschaften e.V. (AWMF-DK) - Association of the Scientific Medical Societies in Germany}

\author{
Frankfurt, 6. November 2021
}

Die Delegiertenkonferenz wurde als Präsenzkonferenz kombiniert mit virtuellem Format technisch einwandfrei unter Einbeziehung einer Abstimmungsplattform durchgeführt.

Nach Begrüßung und stillem Gedenken an den im Mai 2021 verstorbenen langjährigen Präsidenten der AWMF, Herrn Prof. Kreienberg, wurde die Beschlussfähigkeit festgestellt und die Tagesordnung genehmigt. Auch das Protokoll der DK vom 8. Mai 2021 wurde ohne Änderungswünsche genehmigt.

Es folgte der Bericht des stellvertretenden Präsidenten der AWMF, Prof. Treede, mit Darlegung der gesundheitspolitischen Forderungen der AWMF. Die AWMF wird sich in das Lobby-Register des Deutschen Bundestages eintragen, was Fachgesellschaften möglicherweise auch sollten. Zu den Approbationsordnungen (Humanmedizin, Zahnmedizin, Psychotherapie) fanden Diskussionsrunden statt und es wurde dazu am 02.07.2021 eine AWMF-Stellungnahme verfasst. Speziell zur ÄApprO erfolgten Positionierungen der AWMF an die Kultusministerkonferenz (KMK) zur Wissenschaftlichkeit, den
Grundlagenfächern, Patienten in der Lehre, Examina, Famulatur, PJ, Hausärzte, zur Fächerliste, der Innovationsklausel und zur Bedeutung des IMPP. Gegenüber den Bundesländern wurden die Kosten, die KapVO und das Thema Prüfungen angesprochen.

Die Fächerzuordnung im NKLM wurde breit thematisiert mit der Markierung von Lernzielen durch mandatierte Fachvertreter. Hierzu wurde eine Ad hoc Kommission gegründet. Es wurde auf die Mitgliedschaft der AWMF im Council for International Organiziations of Medical Sciences (CIOMS) hingewiesen, wo es in der Vergangenheit wenig Aktivitäten gegeben hatte, jetzt aber die Teilnahme an einer Working Group ansteht. Zu mehreren Fragen gab es Positionspapiere der AWMF, etwa zum Sitz der wissenschaftlichen medizinischen Fachgesellschaften im Gemeinsamen Bundesausschuss (G-BA) und zum Verhältnis der medizinischen Wissenschaft zur Industrie sowie zur Qualitätssicherung wissenschaftlich basierter Fortbildung und Transparenz bei möglichen Interessenkonflikten. Es gab 10 Stellungnahmen der AWMF im Laufe des Jahres 2021 zu verschiedenen Fragen, etwa zur medizinischen Versorgung und zur Bedeutung der STIKO für Impfempfehlungen. Mit dem G-BA gibt es Verhandlungen über eine Vergütung für Stellungnahmen der Fachgesellschaften. Bei den Leitlinien schreitet die Digitalisierung voran (Beratung und Supervision: AWMF-Task Force IT), eine Task Force COVID-19-Leitlinie wurde etabliert, Leitlinien wurden verfasst, deren Implementierung in die Praxis bedarf der Förderung. Zur Förderung von Evidenzrecherchen finden Gespräche der AWMF mit dem BMG und dem IQWiG statt. In der Diskussion wurden die extrem kurzen Fristen für Stellungnahmen gegenüber dem G-BA als unzumutbar kritisiert, zumal qualifizierte Personen gefunden werden müssen, die im Regelfall in ihrer Freizeit und am Wochenende innerhalb weniger Tage eine Stellungnahme formulieren.

Zu den Aufnahmeanträgen von Fachgesellschaften wurde festgestellt, dass die Aufnahmekriterien einer Überarbeitung bedürfen. Aktuell entscheidungsreife Aufnahmeanträge lagen nicht vor, es sind jedoch 11 Anträge in der Bearbeitung.

Bei den Nachwahlen zum Präsidium der AWMF wurde Prof. Treede, Neurophysiologe an der Medizinischen Fakultät Mannheim der Universität Heidelberg, bisheriger Vizepräsident der AWMF und seit 2006 Mitglied im Präsidium der AWMF, zum neuen Präsidenten der AWMF gewählt. Als Vizepräsident erhielt Prof. Zepp, bis 2021 Direktor der Universitätskinderklinik Mainz, die erforderlichen Stimmen. Als weiteres Mitglied des Präsidiums der AWMF wurde Prof. Deinzer, Leiterin des Instituts für Medizinische Psychologie der Universität Gießen, gewählt.

Breiteren Raum nahm die Befassung mit dem Nationalen Kompetenzbasierten Lernzielkatalog Medizin (NKLM) ein. Am 27. April 2021 wurde der NKLM 2.0 veröffentlicht. Die Weiterentwicklung des NKLM, gesteuert vom Medizinischen Fakultätentag (MFT) und der Implementierungsprozess an den Fakultäten mit Veränderungen des Curriculums aufgrund der ÄApproO sind zu leisten. Dazu müssen Lernziele bewertet werden und die Fakultäten müssen Perspektiven entwickeln. Die NKLM-Inhalte können über einen Bewertungstool in der NKLM-Ansichtsplattform bewertet werden, dies bezogen auf die Schwerpunktthemen in den weiteren Entwicklungsphasen des NKLM. Bei 2623 Items (Lernzielen) gab es Empfehlungen pro Fach, auch wenn die Fächerzuordnungen noch ausstehen. Die Empfehlungen sind unverbind- 
lich und als Hilfestellung für die Fakultäten bzw. Studiendekanate gedacht.

Umfassender diskutiert wurde die Frage eines quantifizierbaren Zusatznutzens für das Engagement der Fachgesellschaften v. a. für die Kommission Nutzenbewertung von Arzneimitteln und die Erarbeitung von Stellungnahmen. Dabei wurde verdeutlicht, dass die Arbeit der Fachgesellschaften von großem Nutzen ist und Einfluss hat auf die Nutzen-Bewertung des G-BA und damit auf die Qualität in der Patientenversorgung. In vielen Verfahren der Nutzenbewertung konnten mehrere Fachgesellschaften, in Einzelfall bis zu fünf Fachgesellschaften, ihre Expertise einbringen. Die Einbeziehung der Fachgesellschaften wurde im Jahre 2019 auch im Gesetz für mehr Arzneimittelsicherheit festgeschrieben. In der Praxis gibt es im Vorfeld häufig schon Anfragen zur Vorbereitung von Verfahren der frühen Nutzenbewertung. Im Jahre 2020 gab es ca. 200 Verfahren, im Jahre 2021 können es bis zu 300 Verfahren für die Kommission Arzneimittelsicherheit werden. Für das Beratungsverfahren wurde eine überschaubare Kostenerstattung vereinbart zwischen dem G-BA und der AWMF. Die AWMF hält einen festen Sitz der wissenschaftlichen medizinischen Fachgesellschaften im G-BA für geboten, dies ohne Stimmrecht.

Der Präsident des Bundesamts für Arzneimittel und Medizinprodukte (BfArM), Prof. Broich, berichtete zu aktuellen Themen und Herausforderungen für das BfArM in der Corona-Pandemie mit Modifikationen etwa des Zulassungsverfahrens für Arzneimittel („,Rolling Review“ als Instrument in der Pandemie-Notfallsituation, bislang 16 Verfahren, 6 Verfahren für therapeutische Produkte, 10 Verfahren für Impfstoffe). Für Verfahren mit COVID-19-Bezug wurden Gebühren reduziert. Liefer- und Versorgungsengpässe, z. B. beim Propofol, waren aufgetreten und es ging um Sonderzulassungen von Medizinprodukten (Masken, Tests). Die digitale Transformation im Gesundheitswesen wirkt sich ebenfalls aus auf die Arbeit des BfArM (z. B. Zulassung von Gesundheits-Apps bzw. digitalen Gesundheitsanwendungen; DiGA). Auch die Durchführung klinischer Prüfungen wurde von der Corona-Pandemie beeinflusst (z. B. konnten Patienten Prüfzentren nicht fristgerecht aufsuchen). Internationale Aktivitäten des BfArM u. a. zum Umgang mit EU-Gesundheitsdaten sind in der Entwicklung.

Vorgetragen wurde zum Sachstand bei der Digitalisierung des Leitlinienwissens. Für strukturierte digitale Leitlinien (LL) muss das AWMF-Leitlinienregister angepasst werden mit verbesserter Handhabung von LL nach Überarbeitung des LL-Portals. Die Neugestaltung der Website der AWMF soll mit einfacher und erweiterter Suchfunktion eine bessere Hilfestellung geben, was am Beispiel des kolorektalen Karzinoms dargestellt wird.

Abschließend wurden Chancen und Risiken einer Neuordnung der In-vitro-Diagnostik (IVDR) diskutiert, der Haushaltsplan 2022 vorgestellt und Termine für die Delegiertenkonferenzen der AWMF im Jahre 2022 vorgeschlagen.

\section{R. Dettmeyer}

Gießen

\section{Personalia}

Die Autoren Benjamin Ondruschka, Ugo Da Broi, Cristian Palmiere, Rexson Tse und Jack Garland haben den „Best paper award "des Journals Forensic Science International 2021 NIFS Awards für den Artikel Post mortem tryptase: A review of literature on its use, sampling and interpretation in the investigation of fatal anaphylaxis gewonnen. Die DGRM gratuliert herzlichst.

Termine rechtsmedizinischer Kongresse entnehmen Sie bitte den folgenden Webseiten oder scannen Sie den QR-Code:

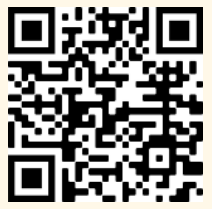

https://www.dgrm.de/tagungen/jahrestagung-dgrm

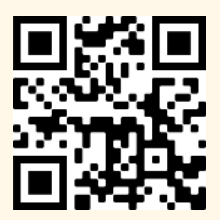

http://www.gmkongresse.de 